\title{
Sneutrino condensate as a candidate for the hot big bang cosmology
}

\author{
Anupam Mazumdar ${ }^{1}$ and Abdel Pérez-Lorenzana ${ }^{2}$ \\ ${ }^{1}$ McGill University, 3600 University Road, Montréal, Québec, H3A 2T8, Canada \\ ${ }^{2}$ Departamento de Física, Centro de Investigación y de Estudios Avanzados del I.P.N., Apartado Postal 14-740, 07000, México, \\ D.F., México
}

(Received 21 June 2004; published 20 October 2004)

\begin{abstract}
If the inflationary paradigm is correct, then it must create conditions for the hot big bang model with all observed matter, baryons, and the seed perturbations for structure formation. In this paper we propose a scenario where the inflaton energy density is dumped into the bulk in a brane world setup, and all the required physical conditions are created by the right-handed neutrino sector within supersymmetry. The scalar component of the right-handed Majorana neutrino is responsible for generating the scale invariant fluctuations in the cosmic microwave background radiation, reheating the Universe at a temperature $T_{\mathrm{rh}} \leq 10^{9} \mathrm{GeV}$ and finally generating the lepton/baryon asymmetry $n_{B} / s \sim 10^{-10}$ with no lepton/baryon-isocurvature fluctuations.
\end{abstract}

DOI: 10.1103/PhysRevD.70.083526

PACS numbers: $98.80 . \mathrm{Cq}, 11.10 . \mathrm{Kk}$

\section{INTRODUCTION AND SUMMARY}

Inflation is the only dynamical mechanism which stretches the quantum fluctuations causally outside the horizon, besides making the Universe flat, homogeneous, and isotropic [1]. The primordial perturbations, which enter the Hubble horizon after the end of inflation, act as a seed for the large scale structures and the fluctuations in the cosmic microwave background radiation [2]. The vacuum dominated inflation leaves the Universe devoid of any entropy; therefore, it is important that any inflationary paradigm must pave the way to a hot big bang cosmology with matching standard model (SM) relativistic degrees of freedom.

It is usually believed that the entropy is generated from the decay of the inflaton energy density; however, it is unfortunate that the inflaton sector remains enigmatic. It is often regarded as a SM gauge singlet and, in many models, an absolute gauge singlet, whose potential is sufficiently flat to provide enough e-foldings of inflation with almost scale invariant density perturbations. In this regard the inflaton potential can be considered as a dark energy ${ }^{1}$ with a yet unanswered puzzle, "how does this unknown sector couple to the SM degrees of freedom?"

Indeed, it is possible that the inflaton does not couple to the SM degrees of freedom at all, as suggested in Refs. [4-9]. In these examples the gauge invariant flat directions of minimal supersymmetric SM (MSSM) (for a review, see [10]) was responsible for reheating the Universe with the MSSM degrees of freedom. In Ref. [7], we explored a similar possibility with a sneutrino condensate; see also Ref. [11].

\footnotetext{
${ }^{1}$ Often in the literature the dark energy is referred to the present acceleration, for which we do not have a good understanding; see [3]. In this paper we regard the primordial inflation as a dark energy sector, mainly because we do not know the inflaton sector at all.
}

Although conventionally one relates the spectrum of the density perturbations to the properties of the inflaton potential, recently it has been realized that this is not a necessary condition for a successful inflationary scenario. In curvaton models the dark energy induces quantum fluctuations in a field whose energy density during inflation is negligible but which may later become dominant [12-15].

On the other hand, the recent developments in the neutrino experiments suggest that the neutrinos have nonvanishing masses. Solar neutrino deficit is better understood if the electron neutrinos oscillate into the muon neutrinos controlled by the squared mass difference, $\Delta m_{\text {solar }}^{2} \sim 7 \times 10^{-5} \mathrm{eV}^{2}$, with a large mixing angle $\tan ^{2} \theta_{\text {solar }} \sim 0.5$ [16], whereas the atmospheric neutrino experiments indicate $\nu_{\mu}-\nu_{\tau}$ oscillations with $\Delta m_{\mathrm{atm}}^{2} \sim$ $2.5 \times 10^{-3} \mathrm{eV}^{2}$ and $\sin ^{2}(2 \theta) \simeq 1$ [17].

If the neutrinos were Dirac particles, unnaturally small Yukawa couplings of the order of one part in $10^{11}$ were required in order to obtain $m_{\nu} \sim 1 \mathrm{eV}$. The most natural explanation arises if the neutrinos are Majorana particles along with the seesaw mechanism that involves large right-handed neutrino masses [18]. One advantage of this mechanism is that the right-handed neutrino mass breaks $L$ (or $B-L$ ) quantum number, which can create a lepton asymmetry. The conversion of the leptonic asymmetry into the baryonic asymmetry, via active SM sphalerons within a range of $10^{12} \mathrm{GeV} \geq T \geq 100 \mathrm{GeV}$, addresses the observed baryon asymmetry [19].

In a supersymmetric theory, which we assume in our analysis, the sneutrino condensate decay can also induce leptogenesis. This scenario, in principle, can be tested by the cosmic microwave background radiation $(\mathrm{CMB})$ via the baryon-isocurvature fluctuations [20]. In our case the sneutrino will decay into the sleptons and the Higgsinos, thus ensuring reheating into the MSSM and the SM degrees of freedom. 
In Refs. [6-8], it was suggested that the inflaton energy density can be deposited away from the observable world within a brane world setup. In brane world models the Universe is assumed to be a four-dimensional (4D) surface (brane) embedded in a bulk of $4+n$ dimensions. The $n$-extra dimensions can be either open or compact (for a review, see [21]). The standard setup assumes that the higher dimensional graviton propagates in the bulk, while the SM gauge fields are stuck on the brane. The bulk geometry could be warped with an anti-de Sitter $(\operatorname{adS})$ metric $[22,23]$. In these scenarios the higher dimensional Planck scale $M_{*}$ can be different from the fourdimensional Planck mass $M_{p}=2.4 \times 10^{18} \mathrm{GeV}$, which are related to each other by the volume of the extra space. When the bulk is adS, it is possible to localize the zero mode of the bulk gravitons as a four-dimensional graviton from the brane point of view.

In the background of an adS geometry, the quasilocalized zero mode of a massive bulk field, at a brane position, has a finite lifetime to leave the brane [24]. It is equally possible that a brane field has an exclusive coupling to the bulk degrees of freedom. In the latter case also the brane field will eventually decay into the bulk modes. Suppose that the inflaton is such a candidate which couples only to the bulk modes; this could happen due to some underlying symmetry which prevents it from having renormalizable and nonrenormalizable couplings to the MSSM matter fields, in which case the question arises: how would the Universe evolve after inflation?

Inflation excites any light scalar fields. Therefore, any light degrees of freedom, lighter than the Hubble expansion rate, will obtain quantum fluctuations. Such light fields act as a homogeneous condensate during inflation; see [10]. The dynamics of the sneutrino fields can be almost frozen if their masses are lighter than the Hubble expansion rate. Obviously, the dynamics of the lighter ones will prevail over the others. The fluctuation in the lightest field is isocurvature in nature during inflation; however, after inflation the lightest sneutrino condensate dominates the energy density. During its decay the sneutrino not only generates lepton asymmetry and the MSSM relativistic degrees of freedom but also converts isocurvature perturbations into adiabatic perturbations. It is therefore the sneutrino condensate which leads to the hot big bang cosmology and not the inflaton; the inflaton sector remains the darkest side of the Universe.

We begin our discussion with an inflaton decaying into the bulk modes. We then describe the origin of light and heavy Majorana neutrino masses. We discuss the dynamics of the sneutrino condensate and explain various implications.

\section{REHEATING THE BULK MODES}

For an example, we work in a five-dimensional (5D) brane world setup, where the bulk is infinitely large with a warped metric

$$
d s^{2}=g(z)_{M N} d x^{M} d x^{N}=\omega^{2}(z) g_{\mu \nu} d x^{\mu} d x^{\nu}-d z^{2},
$$

where $g_{\mu \nu}$ is the four-dimensional brane metric, with all the SM gauge degrees of freedom fixed to the brane located at $z=0$ [22]. The warp factor in the metric has a form $\omega(z)=e^{-\kappa|z|}$, where $\kappa$ is a constant that relates the true gravitational coupling constant of the fivedimensional theory, $G_{5}$, to the low energy effective four-dimensional Newton's constant via $G_{N}=\kappa G_{5}$. In this theory the Planck scale, $M_{P}=\left(8 \pi G_{N}\right)^{-1 / 2}=2.4 \times$ $10^{18} \mathrm{GeV}$, is derived from the fundamental gravitational scale, $M_{*}=\left(8 \pi G_{5}\right)^{-1 / 3}$, through the relationship

$$
M_{*}^{3}=\kappa M_{P}^{2} \text {. }
$$

Here onwards we take $\kappa$, and therefore $M_{*}$, to be close to the Planck scale. ${ }^{2}$ In this setup the brane has a tension $\sigma=6 \kappa M_{*}^{3}$, whereas the bulk is an adS slice with a negative cosmological constant $\Lambda=-\sigma^{2} / 6 M_{*}^{3}$ [23].

For the purpose of illustration, let us assume that the inflaton is a true 4D brane field which is homogeneously distributed and dominating the energy density. The modified Friedmann equation has a quadratic dependence on the brane density $\rho$; see [25-28]. From the point of view of an effective field theory on the brane, the Hubble expansion rate $H=\dot{a} / a$, where $a$ is the scale factor of the four-dimensional Universe, is determined by

$$
H^{2}=\frac{1}{3 M_{P}^{2}} \rho\left(1+\frac{\rho}{2 \sigma}\right)+\frac{\sigma}{2 M_{p}^{2}}\left(\frac{a_{h}}{a}\right)^{4} .
$$

The last term is an interesting one, which needs some explanation. The adS has a horizon; in order to see that, let us assume that a point particle is traveling in the bulk; it has a momentum along the fifth component. Although the particle is moving towards infinity, it actually takes a finite proper time $\tau=(\pi / 2 \kappa)$ to reach the end of the space; see [29]. Hence, $z=\infty$ actually acts as a particle horizon. The boundary conditions at the horizon are imposed such that nothing comes in from behind the horizon.

The four-dimensional Poincaré invariance ensures that the coordinate four momentum $p_{\mu}$ coincides with the physical momentum on the brane, but from the point of view of an observer, away from the brane, $z \neq 0$, the fourdimensional momentum gets blueshifted; this means that the modes which are softer on the brane become harder away from the brane. This leads to a conjecture that a black hole might form at the horizon of the adS space $[27,28]$. In the above expression, $a_{h}$ is understood as a black hole horizon [27]. It is always compared with the scale factor $a(t)$ on the brane. The second term is a correction due to the brane position breaking the $5 \mathrm{D}$

\footnotetext{
${ }^{2}$ For instance, $M_{*} \sim 0.5 M_{p}$. This gives $\kappa \sim 0.1 M_{p}$.
} 
Lorentz invariance, such that the brane tension is acting as a source term in 5D.

The main point is to note that the standard Hubble relationship $H=\sqrt{\rho / 3 M_{P}}$ follows only for small densities compared to the brane tension $\rho \ll \sigma[25,26]$, and the contribution from the last term is subdominant as long as the scale factor on the brane position, $a \gg a_{h}$, is greater than the black hole horizon [28]. With these assumptions thermal history of the Universe follows similar to the standard cosmology [30]. We will always be working at energy scales below the brane tension, therefore, with the standard Friedmann equation.

Let us suppose that the inflaton and the bulk fields carry some global quantum number, while the brane degrees of freedom do not. In such a case the inflaton energy will be radiated away into the bulk after the end of inflation in the form of Kaluza-Klein (KK) modes, from the four-dimensional point of view. These bulk modes are carrying the momentum along the fifth dimension; therefore, they will simply fly away towards infinity. For the purpose of illustration, let us now assume that the brane inflaton field $\phi$ couples to some bulk scalar field through the coupling ${ }^{3}$

$$
\sqrt{g(z)} \xi \phi(x) \varphi(x, z) \varphi(x, z) \delta(z),
$$

where $\xi$ is a dimensionless coupling constant. This coupling induces a large decay rate for the inflaton to the bulk modes. Our scenario can be thought of as a hot radiating plate cooling down by emitting its energy into its cold surroundings [31]. To estimate how efficient this process is, let us first comment on the KK decomposition of the bulk field. Consider the action for a bulk scalar field $\varphi$, of mass $\mu$, in the background metric Eq. (2.1),

$$
S_{\chi}=\int d^{4} x d z \sqrt{g^{(5)}}\left(\frac{1}{2} g^{M N} \partial_{M} \varphi \partial_{N} \varphi-\frac{1}{2} \mu^{2} \varphi^{2}\right) \text {. }
$$

The equation of motion for the bulk scalar field then reads as

$$
\left[-\partial_{z}^{2}+4 \kappa \operatorname{sgn}(z) \partial_{z}+\mu^{2}+\omega^{-2}(z) \partial^{\mu} \partial_{\mu}\right] \varphi(x, z)=0 .
$$

Since the fifth dimension is not compact, the KK spectrum is continuous, and the scalar field can be expanded as

$$
\varphi(x, z)=\int \frac{d m}{\kappa} \varphi_{m}(x) \chi(z ; m),
$$

where the KK wave function $\chi(z ; m)$ satisfies the reduced field equation

$$
\left[-\partial_{z}^{2}+4 \kappa \operatorname{sgn}(z) \partial_{z}+\mu^{2}-\omega^{-2}(z) m^{2}\right] \chi(z ; m)=0 .
$$

The normalization condition is given by $\int d z \omega^{2}(z) \times$

\footnotetext{
${ }^{3}$ The inflaton coupling to the fermions was already studied in Ref. [7]; here we describe the bosonic interaction.
}

$\chi(z ; m) \chi\left(z ; m^{\prime}\right)=\kappa \delta\left(m-m^{\prime}\right)$, while the boundary condition on the brane is $\partial_{z} \chi(z=0 ; m)=0$.

In the above equations, $m^{2}=p^{\mu} p_{\mu}$ is the fourdimensional mass. The general solution to the above equation is given in terms of the Bessel functions of index $\nu=\sqrt{4+\mu^{2} / \kappa^{2}}[24,32]$

$$
\chi(z ; m)=\frac{1}{N(m) \omega^{2}(z)}\left[J_{\nu}\left(\frac{m}{\kappa \omega(z)}\right)+A(m) Y_{\nu}\left(\frac{m}{\kappa \omega(z)}\right)\right],
$$

where $N$ is the normalization factor, and the coefficient $A(m)$ is given by

$$
A(m)=-\frac{2 J_{\nu}(m / \kappa)+(m / \kappa) J_{\nu}^{\prime}(m / \kappa)}{2 Y_{\nu}(m / \kappa)+(m / \kappa) Y_{\nu}^{\prime}(m / \kappa)} .
$$

In the limiting case, where $\mu, m \ll \kappa$, one can take $\nu=2$ in the above expressions to approximate the KK wave function evaluated at the brane position by

$$
\chi(0 ; m) \approx \sqrt{\frac{m}{2}} .
$$

Next we use these results to evaluate the decay rate of the inflaton into the bulk modes. From Eq. (2.4), we can read the effective coupling of the inflaton,

$$
\xi\left[\chi(0 ; m) \chi\left(0 ; m^{\prime}\right)\right] \phi(x) \varphi_{m}(x) \varphi_{m^{\prime}}(x) .
$$

Note that this effective coupling constant is essentially proportional to the geometric mean of the product of the KK masses, $\sqrt{\mathrm{mm}^{\prime}}$. In our case the inflaton will preferably decay into those KK modes with the largest possible fifth momentum allowed by the kinematics. These excited modes will move away from the brane towards infinity.

The total decay rate is estimated in the low energy limit, where $\mu, m_{\phi} \ll \kappa$, as

$$
\begin{aligned}
\Gamma_{\phi} & =\int_{0}^{m_{\phi}} \int_{0}^{\sqrt{m_{\phi}^{2}-m^{2}}} \frac{d m}{\kappa} \frac{d m^{\prime}}{\kappa} \xi^{2} \frac{\left[\chi(0, m) \chi\left(0, m^{\prime}\right)\right]^{2}}{m_{\phi}} \\
& \approx \frac{\xi^{2}}{32}\left(\frac{m_{\phi}}{\kappa}\right)^{2} m_{\phi} .
\end{aligned}
$$

The above expression clearly shows that, for a sufficiently heavy inflaton, the decay rate to the bulk KK modes is quite efficient.

Let us now briefly discuss the fate of the energy density which is stored in the bulk modes. From the fourdimensional point of view one can track the inflaton energy density from the Friedmann equation, Eq. (2.3). The last contribution in the right-hand side actually accounts for a dark radiation, which depends on the redshift factor $\left(a_{h} / a\right)^{4}$. In fact, this ratio becomes exceedingly small during the exponential expansion of the Universe compared to the other terms in the Friedmann equation. We can estimate this ratio by assuming that at the beginning of inflation the energy density is stored in the vac- 
uum energy density of the inflaton. For the time being, we also assume that there is no other source for energy density; in that case, from the brane observer point of view, the largest scale could be the Compton wavelength corresponding to the inflaton mass scale, which is at most $a_{h} \sim[V(\phi)]^{-1 / 4}$. For typical values, $V(\phi)^{-1 / 4} \sim$ $10^{-16}(\mathrm{GeV})^{-1} \sim 10^{-32} \mathrm{~m}$, we find $a_{h}$ is negligible compared to the present size of the Hubble horizon. The ratio $a_{h} / a \ll 1$ becomes small by the time inflation comes to an end.

In fact, in our case, we can roughly estimate the dark radiation ${ }^{4}$ contribution to the present day. We estimate the rate of loss of energy

$$
\frac{\Delta \dot{\rho}}{\rho}=\mathcal{O}(1) \Gamma_{\phi} .
$$

The order one coefficient takes into account the relativistic degrees of freedom of the inflaton decay products. At sufficient late times, $1 / H \gg 1 / \kappa$, or $\rho \ll \max \{V(\phi), \sigma\}$, the total contribution to the dark radiation will be roughly given by the loss in the energy density from the brane, e.g., $\Delta \dot{\rho}+\Delta \dot{\rho}_{d}=0$ [28],

$$
\Omega_{d}=\frac{\rho_{d}}{\rho_{d}+\rho} \approx \int_{\tau_{1}}^{\infty} d \tau\left(-\frac{\Delta \dot{\rho}}{\rho}\right) .
$$

We can estimate the integral and the final answer is given by

$$
\Omega_{d} \approx \frac{\xi^{2} \pi}{64}\left(\frac{m_{\phi}}{\kappa}\right)^{3} .
$$

For $\kappa \gg m_{\phi}$, the dark radiation contribution is negligible.

There are other notable effects of the inflaton quanta escaping into the bulk. Because of causality they will leave a wake in the form of gravitational fluctuations. In fact, a particle moving with a trajectory perpendicular to the brane follows a world line $z(t)=\ln \left(1+\kappa^{2} t^{2}\right) / 2 \kappa$. As the particle leaves the brane, it produces a gravitational wave, which dies away as $\sim r / t$, for distances $r$ within the light cone; see, for instance, Ref. [24] and the last reference in [29]. These effects will not be imprinted on the cosmic background, because these are subhorizon effects which are diluted away in the course of expansion. For our purposes we will now continue working with the standard cosmology with $H \propto \rho^{1 / 2} / M_{p}$.

\section{NEUTRINO MASSES}

Neutrino masses can well have their origin in four dimensions. Let us assume a simple superpotential for the right-handed Majorana neutrinos,

$$
W=\frac{1}{2} M_{N} \mathbf{N N}+h \mathbf{N L} \mathbf{H}_{\mathbf{u}},
$$

\footnotetext{
${ }^{4}$ This should not be confused by the primordial dark energy contribution we referred to as the unknown sector of the inflaton energy density.
}

where $\mathbf{N}, \mathbf{L}, \mathbf{H}_{\mathbf{u}}$ stand for the neutrino, the lepton, and the Higgs doublet, respectively; $M_{N}, h$ are $3 \times 3$ mass and the Yukawa matrices, respectively. We have assigned an odd $R$ parity for the right-handed (s)neutrinos, which prohibits $(\mathbf{N})^{3}$ term in the superpotential. The only possible interactions are through the Yukawa matrix, $h_{i j}, i, j=1,2,3$, with the SM lepton and the Higgs fields. The origin of the mass term $M_{N}$ could be due to $\mathrm{SO}(10)$ broken down to $\mathrm{SU}(5)$ through the condensation of the SU(5) singlet components in $(126, \overline{126})$ representation, for instance. However, the crucial point is that below the $\mathrm{SO}(10)$ breaking scale, the $\mathrm{D}$ term decouples from the potential of the sneutrino, $\tilde{N}$, which restricts the vacuum expectation value (vev) as $\tilde{N} \leq M_{\text {GUT }}$. Among other possibilities, we can also think of breaking a simple group for, e.g., $\mathrm{U}(1)_{B-L}$, which can give rise to the masses of the neutrinos.

Irrespective of the origin of the right-handed neutrino mass term, we can write down a potential barring any nonrenormalizable interactions and soft supersymmetry breaking $A$ terms,

$$
V \sim M_{N}^{2} \tilde{N}^{2} .
$$

Nonrenormalizable terms including higher powers of $N$ fields can always be removed by the addition of $R$ symmetry, which we assume, whereas the mass scale of the soft supersymmetry breaking terms is typically small (about a few $\mathrm{TeV}$ ) compared to the expected mass scale of the right-handed neutrinos, required to be around $10^{10} \mathrm{GeV}$ by the seesaw in our case. For simplicity we always assume a diagonal basis for the right-handed neutrino mass matrix. The left-handed neutrinos obtain masses via a seesaw mechanism [18]

$$
m_{\nu} \sim m_{D}^{T} M_{N}^{-1} m_{D},
$$

where $m_{D}$ is the Dirac mass matrix.

For simplicity, we may also assume that the lightest sneutrino mass is smaller than the Hubble expansion rate during inflation. We also assume that its largest Yukawa coupling is that of the $\tau$ doublet, $h_{1,3} \sim 10^{-4}-10^{-5}$. Note that by taking $M_{N} \sim 10^{10} \mathrm{GeV}$, with $M_{N_{2,3}} \gg M_{N_{1}}$, we can obtain a small electron-tau neutrino mixing in the left-handed sector, as seems to be required by the small $\theta_{13}$ angle in the neutrino experiments, provided there is a natural hierarchy in the neutrino masses.

A possible texture that may give the desired bilarge mixing in the left-handed neutrino sector can be given by $[33,34]$

$$
m_{\nu} \sim m_{0}\left(\begin{array}{ccc}
\lambda & \lambda & \lambda \\
\lambda & 1+\lambda & 1 \\
\lambda & 1 & 1
\end{array}\right),
$$

where $m_{0} \approx \sqrt{\delta m_{\mathrm{atm}}^{2}}$ and $\lambda$ is a small parameter. This texture gives hierarchical neutrino masses, with mass eigenvalues $m_{3} \approx m_{0}$ consistent with atmospheric, and $m_{2} \approx \lambda m_{0}$, for solar oscillations, with a smaller $m_{1}$. We 
can also obtain $\tan \theta_{\text {atm }} \approx 1$ and a large mixing angle required for explaining the solar neutrino oscillations. Although our intention is not to present a model for the neutrino masses in this paper, it is nice to see that the physics involved in our discussion seems to provide a consistent picture with the minimal requirement on some models for the neutrino masses and mixings.

\section{DYNAMICS AND DENSITY PERTURBATIONS FROM $\tilde{N}_{1}$}

During vacuum dominated inflation, there is always quantum fluctuation in spite of the fact that inflation wipes out all the inhomogeneities. A massless bosonic field can have Brownian jumps of order $\sim H / 2 \pi$ [35]. However, during one e-folding of inflation the field becomes homogeneous in a Hubble patch and therefore acts as a cosmic condensate. These jumps facilitate the lighter fields to obtain nonvanishing vacuum expectation values during inflation. The maximum amplitude of the light field can be determined by $V(\tilde{N}) \leq V(\phi)_{\text {inf }}$, such that the inflaton energy density remains dominating. We denote the lightest right-handed sneutrino by $\tilde{N}_{1}$, assuming that it is lighter than the Hubble expansion rate during inflation.

We are interested in tracking the classical and quantum evolution of $\tilde{N}_{1}$. In fact, $\tilde{N}_{1}$ acts as a curvaton field [1214]. During inflation the energy density of the curvaton field is subdominant. Consequently, quantum fluctuations of this field lead to the isocurvature perturbations. Current CMB measurements tell us that the density perturbations are mainly of the adiabatic type [2]. Hence, for the curvaton scenario to work, isocurvature perturbations have to be converted into adiabatic ones. Such a conversion takes place when the contribution of the curvaton energy density, $\rho_{\tilde{N}_{1}}$, to the total energy density in the universe grows, i.e., with an increase of

$$
r=\frac{3 \rho_{\tilde{N}_{1}}}{4 \rho_{\gamma}+3 \rho_{\tilde{N}_{1}}} .
$$

Here $\rho_{\gamma}$ is the energy in the radiation bath from the inflaton decay. Non-Gaussianity of the produced perturbation requires the curvaton to contribute more than $1 \%$ to the energy density of the Universe at the time of decay; that is, $r_{\mathrm{dec}}>0.01$, required by the present observation [2]. In our case the inflaton energy density does not reheat the Universe; therefore, the above ratio $r=1$ is naturally satisfied, suggesting that there is no possible signature of primordial non-Gaussianity in CMB fluctuations. This should be considered as one of our predictions.

The long wavelength fluctuations in the sneutrino field leave the Hubble horizon; these perturbations can be defined on a finite energy density hypersurface foliated in a coordinate system, such that the metric perturbation is $\zeta$ and the metric (for a detailed discussion on cosmological density perturbations, see [36]) is given by

$$
d s^{2}=a^{2}(t)(1+2 \zeta) \delta_{i j} d x^{i} d x^{j},
$$

where $a$ is the scale factor. The time evolution of the curvature perturbation, $\zeta$, on scales larger than the size of the horizon is given by [37-39]

$$
\dot{\zeta}=-\frac{H}{\rho+P} \delta P_{\text {nad }}
$$

where $P_{\text {nad }} \equiv \delta P-c_{s}^{2} \delta \rho$ is the nonadiabatic pressure perturbation. The adiabatic sound speed is $c_{s}^{2}=\dot{P} / \dot{\rho}$, where $P$ and $\rho$ are the total pressure and energy density, respectively. For a single field $\delta P_{\text {nad }}=0$; therefore, on large scales the curvature perturbation is pure adiabatic in nature with $\zeta=$ constant. This is not true in the presence of many fields, because relative pressure perturbations between fields can give nonzero contribution to $\delta P_{\text {nad }}$.

The unperturbed and the perturbed equations of motion for the sneutrino are given by

$$
\begin{gathered}
\ddot{\tilde{N}}{ }_{1}+3 H \dot{\tilde{N}}_{1}+M_{N, 1}^{2} N_{1}=0, \\
\delta \ddot{\tilde{N}}_{1 k}+3 H \dot{\delta} \tilde{N}_{1 k}+\frac{k^{2}}{a^{2}} \delta \tilde{N}_{1 k}+M_{N, 1}^{2} \delta \tilde{N}_{1 k}=0 .
\end{gathered}
$$

In the limit when $M_{N, 1}^{2} \ll H^{2}$, the perturbations in $\tilde{N}_{1}$ are Gaussian, and the spectrum is given by

$$
\mathcal{P}_{N_{1}}^{1 / 2}=\frac{H_{*}}{2 \pi},
$$

where $*$ denotes the epoch of horizon exit $k=a_{*} H_{*}$.

After the energy density stored in the sneutrino condensate overtakes the redshifted inflaton energy density, the sneutrino condensate starts oscillating. During this epoch the mean squared fluctuations in the sneutrino condensate follows:

$$
\left\langle\left(\delta N_{1}\right)^{2}\right\rangle=\int_{k_{\min }}^{k_{\max }} \mathcal{P}_{N_{1}}(k) \frac{d k}{k} \sim \frac{H_{*}^{2}}{4 \pi^{2}} \ln \left(\frac{k_{\max }}{k_{\min }}\right) \sim \frac{H_{*}^{2}}{4 \pi^{2}},
$$

where $k_{\max } \sim(\tilde{a} \tilde{H})$ and tilde denotes the subhorizon redshifted modes and $k_{\min }=a_{0} H_{0}$, where 0 corresponds to the present values. The field perturbation is then given by

$$
\delta=2 \frac{\delta N_{1}}{N_{1}}=\frac{H_{*}}{\pi N_{1 *}},
$$

when $N_{1, *} \sim 10^{5} \times H_{*}$, in order to match the amplitude for the CMB fluctuations. On the other hand, we require the lightest sneutrino mass should be smaller than the Hubble expansion rate during inflation,

$$
V^{\prime \prime}\left(\tilde{N}_{*}\right)=M_{N_{1}}^{2} \sim \alpha^{2} H_{*}^{2},
$$

where $\alpha<1$, while the scale of inflation is given by $V_{I}^{1 / 4} \sim\left(H_{*} M_{p}\right)^{1 / 2}$.

The amplitude of the fluctuation of the sneutrino field created during inflation will be imprinted on radiation when the condensate decays. The total curvature perturbation is then given by [13] 


$$
\zeta=\zeta_{N_{1}}=-H \frac{\delta \rho_{N_{1}}}{\rho_{N_{1}}}=\frac{1}{3} \delta
$$

and $\mathcal{P}_{\zeta}^{1 / 2} \approx H_{*} / N_{1 *}$. The spectral index of microwave temperature perturbations can then be evaluated as [40]

$$
n_{s}-1=\frac{d \ln \mathcal{P}_{N_{1}}}{d \ln k}=2 \frac{\dot{H}_{*}}{H_{*}^{2}}+\frac{2}{3} \frac{M_{N_{1}}^{2}}{H_{*}^{2}} \sim \frac{2}{3} \alpha^{2} .
$$

For $\alpha \sim 10^{-1}$, we find the spectral index is fairly close to 1 , which is consistent with the recent Wilkinson Microwave Anisotropy Probe observation of $n_{s}=0.99 \pm$ 0.04 [41]. The only assumption is that the Hubble expansion rate during inflation is almost constant; therefore, the first term in the spectral index is negligible while the main contribution comes from the curvature of the sneutrino field.

Since the sneutrino field is dominating the energy density while decaying into sleptons and Higgsinos, there are no residual isocurvature perturbations left over; all of them are converted into adiabatic ones. Later we will comment on the baryon-isocurvature perturbations.

\section{REHEAT TEMPERATURE}

In our case it is the coherent oscillations of the sneutrino field which lead to reheating. For the details of sneutrino reheating, we refer the reader to Ref. [42]. In our case the perturbative decay rate of the lightest sneutrino condensate can be estimated by $\Gamma \sim\left|h_{13}\right|^{2} M_{N_{1}} / 4 \pi$, and the reheat temperature, $T_{\mathrm{rh}} \sim 0.1 \sqrt{\Gamma M_{p}}$,

$$
T_{\text {rh }} \sim 10^{9}\left(\frac{h_{13}}{10^{-4}}\right)\left(\frac{M_{N_{1}}}{10^{10}}\right)^{1 / 2} \mathrm{GeV} .
$$

In order to avoid the gravitino problem, the reheat temperature of the Universe must be $10^{9} \mathrm{GeV}$ [43]. In order to satisfy this bound, the lightest right-handed sneutrino (neutrino) mass must be around $10^{10} \mathrm{GeV}$. This constrains the scale of inflation to be $H_{*} \sim 10^{10} / \alpha \mathrm{GeV}$. If $\alpha \sim 10^{-1}$, then $H_{*} \sim 10^{11} \mathrm{GeV}$ and the initial sneutrino vev, $\left\langle\tilde{N}_{1}\right\rangle \sim 10^{16} \mathrm{GeV}$, which is close to the grand unification scale.

The above numbers are encouraging, because we can nail down the mass of the lightest right-handed neutrino and the initial vev for the sneutrino field. The latter may be indicative that the origin of the masses to the righthanded neutrinos is linked to the grand unified theories (GUT), as, for instance, SO(10) GUT models. It would be interesting to further explore this possibility. Further note that the sneutrino vev is smaller than the $\mathrm{SO}(10)$ breaking scale, therefore justifying our approximation to ignore the D-term contributions in the sneutrino superpotential; see Eq. (3.1). A further comment on the neutrino masses is that the right-handed neutrino mass scale might be different than the GUT scale, provided it originates via non- renormalizable operators, as happens in many $\mathrm{SO}(10)$ constructions.

\section{BARYOGENESIS}

Let us discuss baryogenesis. The sneutrino decay also induces lepton asymmetry due to the $C P$ violation. Net $C P$ asymmetry in our scenario can be calculated by computing the interference between the tree-level and the one-loop diagrams of $\tilde{N}$ going into leptons, $\ell_{j} \tilde{H}_{u}$ (see Fig. 1), and into antisleptons, $\tilde{\ell}_{j}^{*} H_{u}^{*}$ (see Fig. 2), where $j=1,2,3$ flavors $[19,44]$, including the contribution from the wave function mixing diagrams such as those depicted in Fig. 3 [45]. Notice that typical one-loop diagrams that involve quartic scalar couplings do appear from $\mathrm{F}$ terms of the potential; however, they do not contribute to $C P$ asymmetry, although they are needed to cancel the quadratic anomalies.

The $C P$ asymmetry in each decay channel for $X$ (where $\left.X=\ell_{j} \tilde{H}_{u} ; \tilde{\ell}_{j}^{*} H_{u}^{*}\right)$ is defined as

$$
\epsilon_{X} \equiv \frac{\Gamma_{\tilde{N}^{*} X}-\Gamma_{\tilde{N} \bar{X}}}{\Gamma_{\tilde{N}^{*} X}+\Gamma_{\tilde{N} \bar{X}}}
$$

A direct computation of the total $C P$ asymmetry gives [45]

$$
\boldsymbol{\epsilon}=-\frac{1}{4 \pi} \sum_{k \neq 1}\left[\left(\frac{M_{N_{k}}}{M_{N_{1}}}\right) \ln \left(\frac{M_{N_{1}}^{2}}{M_{N_{k}}}+1\right)+2 \frac{M_{N_{1}} M_{N_{k}}}{M_{N_{k}}^{2}-M_{N_{1}}^{2}}\right] I_{k 1},
$$

arising from the vertex and the wave function $C P$ violating diagrams, respectively, where

$$
I_{k 1}=\frac{\operatorname{Im}\left(h_{1 j} h_{1 k} h_{l j}^{*} h_{l k}^{*}\right)}{h_{1 j} h_{1 j}^{*}} .
$$

In the limit of hierarchical right-handed masses, $M_{N_{1}} \ll$ $M_{N_{2,3}}$, which we are considering, we obtain a simplified expression for the $C P$ asymmetry [45]

$$
\epsilon=-\frac{3}{4 \pi} \sum_{k \neq 1}\left(\frac{M_{N_{1}}}{M_{N_{k}}}\right) I_{k 1} \text {. }
$$

The suppression factor, $M_{N_{1}} / M_{N_{k}}$, implies that small $C P$ asymmetry is likely to be produced.

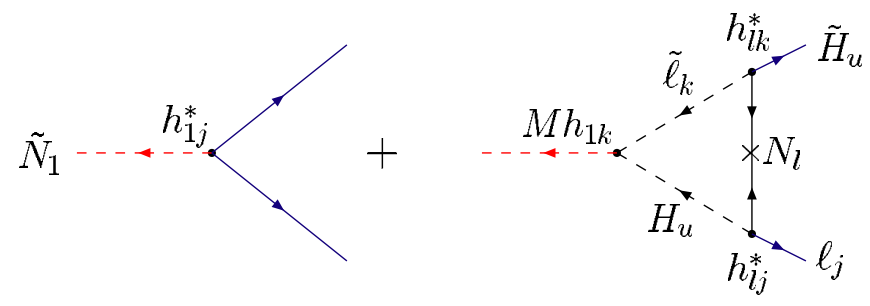

FIG. 1 (color online). Tree-level and one-loop diagrams for the $\tilde{N}_{1}$ decaying into lepton and Higgsino, $\ell_{j} \tilde{H}_{u}$. 

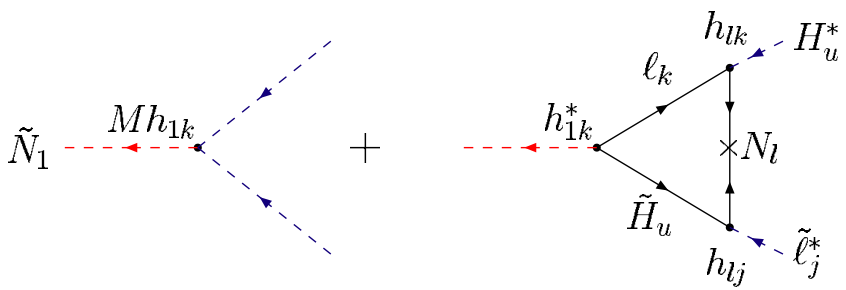

FIG. 2 (color online). Tree-level and one-loop diagrams for the decay of $\tilde{N}_{1}$ into antislepton and Higgs, $\tilde{\ell}_{j}^{*} H_{u}^{*}$.

Now we can predict the left-handed neutrino mass scale with the help of seesaw formula, within our hypothesis of $h_{33}$ dominance, $m_{\nu}=\left|h_{33}\right|^{2} \sin ^{2} \beta /\left(2 \sqrt{2} G_{F} M_{N_{3}}\right)$, where $G_{F}$ is the Fermi constant and $\tan \beta=\left\langle H_{u}\right\rangle /\left\langle H_{d}\right\rangle$. If we take $\left|h_{33}\right| \sim 10^{-1}$ with a typical $\tan \beta \sim 10$ and $M_{N_{3}} \sim 7 \times 10^{13} \mathrm{GeV}$, we obtain $m_{3} \sim 0.04 \mathrm{eV}$, which is already around the expected mass scale for the neutrinos in the case of hierarchical neutrino masses.

The lepton asymmetry can be estimated from the Boltzmann equation,

$$
\dot{n}_{L}+3 H n_{L}=\epsilon \Gamma \frac{\rho_{N_{1}}}{M}-\frac{8}{23} \frac{G_{F}^{2} m_{\nu}^{2}}{\sin ^{4} \beta} T^{3} n_{L} .
$$

The integration becomes simple, especially since we can neglect the rescattering rate of produced leptons which is proportional to the squared mass of the neutrino. The lepton asymmetry is finally converted by the SM sphalerons into the baryons with a net baryon number to the entropy, which is given by $[44,45]$

$$
\frac{n_{B}}{s} \sim \epsilon \frac{6}{23} \frac{T_{\mathrm{rh}}}{M_{N_{1}}} .
$$

The factor $T_{\mathrm{rh}} / M_{N_{1}}$ arises due to the entropy generation from the decay of the condensate. We note that, with $\epsilon \sim$ $10^{-8}$, we can easily generate baryon asymmetry of the order of one part in $10^{10}$, for $T_{\mathrm{rh}} \sim 10^{9} \mathrm{GeV}$ and $M_{N_{1}} \sim$ $10^{10} \mathrm{GeV}$. The actual prediction is an interplay between $T_{\mathrm{rh}}, M_{N_{1}}$, and $\epsilon$. Our analysis seems to favor a rather small value for $\epsilon$.

The fluctuations in the reheat temperature also lead to fluctuations in the baryon asymmetry, $\delta\left(n_{B} / s\right) \propto \delta T_{\mathrm{rh}}$. However, in our case these fluctuations are adiabatic in nature. There are no residual baryon-isocurvature fluctuations generated during the sneutrino decay, because the condensate dominates the energy density while decaying, therefore converting all its isocurvature fluctuations into adiabatic ones. In our simple setup we predict $S_{B}=0$.

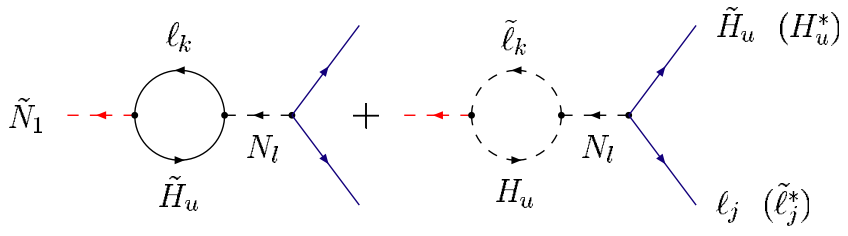

FIG. 3 (color online). One-loop-type diagrams that contribute to wave function mixing for $\tilde{N}_{1}$ decaying into $\ell_{j} \tilde{H}_{u}$. Similar diagrams exist for the decay into $\tilde{\ell}_{j}^{*} H_{u}^{*}$, with the corresponding exchange of external lines as indicated.

This should be taken as a robust claim which can be ruled out very easily from future microwave background anisotropy measurements.

\section{CONCLUSION}

As far as the present state of the art goes, the origin of the inflaton, its potential, and its couplings to the SM are unknown. For various reasons the inflaton is often treated as a gauge singlet; on the other hand, we also often "assume" that the inflaton energy density is dumped into the SM or MSSM degrees of freedom. Here we do not make this assumption; rather we examine what would happen if the inflaton energy density were not to reheat the MSSM (SM) degrees of freedom.

In this regard we separate the two sectors, one of which is responsible for inflation and the other is responsible for generating density perturbations, reheating the Universe with the MSSM degrees of freedom, and creating the observed baryon asymmetry. Our main observation is that there is no logical necessity for the inflaton energy density to be responsible for generating the hot big bang cosmology. The entire thermal history of the Universe can be created from the decay of the sneutrino condensate. It is quite interesting to see that we obtain the right amplitude for the density perturbations, the neutrino mass scale, the reheat temperature below the gravitino overproduction bound, and the observed baryon asymmetry without much fine tuning. An important input in the whole setup is the sneutrino vev, which appears to be close to the grand unification scale.

\section{ACKNOWLEDGMENTS}

We thank Kari Enqvist, James Cline, and Horace Stoica for fruitful discussions. A. P.-L.'s work is supported in part by CONACyT, México, under Grant No. J44596-F.
[1] A. H. Guth, Phys. Rev. D 23, 347 (1981).

[2] C. L. Bennett. et al., Astrophys. J. Suppl. Ser. 148, 1 (2003).
[3] S. M. Carroll, astro-ph/0310342.

[4] K. Enqvist, S. Kasuya, and A. Mazumdar, Phys. Rev. Lett. 90, 091302 (2003). 
[5] K. Enqvist, A. Jokinen, S. Kasuya, and A. Mazumdar, Phys. Rev. D 68, 103507 (2003).

[6] K. Enqvist, S. Kasuya, and A. Mazumdar, Phys. Rev. Lett. 93, 061301 (2004).

[7] A. Mazumdar and A. Pérez-Lorenzana, Phys. Rev. Lett. 92, 251301 (2004).

[8] K. Enqvist, A. Mazumdar, and A. Pérez-Lorenzana, hepth/0403044.

[9] R. Brandenberger and A. Mazumdar, J. Cosmol. Astropart. Phys. 08 (2004) 015.

[10] K. Enqvist and A. Mazumdar, Phys. Rep. 380, 99 (2003).

[11] J. McDonald, Phys. Rev. D 70, 063520 (2004).

[12] K. Enqvist and M.S. Sloth, Nucl. Phys. B626, 395 (2002).

[13] D. H. Lyth and D. Wands, Phys. Lett. B 524, 5 (2002).

[14] T. Moroi and T. Takahashi, Phys. Lett. B 522, 215 (2001); 539, 303(E) (2002).

[15] D. H. Lyth, C. Ungarelli, and D. Wands, Phys. Rev. D 67, 023503 (2003); N. Bartolo and A. R. Liddle, Phys. Rev. D 65, 121301 (2003); A. Hebecker, J. March-Russell, and T. Yanagida, Phys. Lett. B 552, 229 (2003); R. Hofmann, hep-ph/0208267; K. Dimopoulos and D. H. Lyth, Phys. Rev. D 69, 123509 (2004); T. Moroi and H. Murayama, Phys. Lett. B 553, 126 (2003); M. Bastero-Gil, V. Di Clemente, and S.F. King, Phys. Rev. D 67, 103516 (2003); K. A. Malik, D. Wands, and C. Ungarelli, Phys. Rev. D 67, 063516 (2003); M. Postma, Phys. Rev. D 67, 063518 (2003); C. Gordon and A. Lewis, Phys. Rev. D 67, 123513 (2003); B. Feng and M. z. Li, Phys. Lett. B 564, 169 (2003); K. Dimopoulos, D. H. Lyth, A. Notari, and A. Riotto, J. High Energy Phys. 07 (2003) 053; A. R. Liddle and L. A. Urena-Lopez, Phys. Rev. D 68, 043517 (2003); J. McDonald, Phys. Rev. D 68, 043503 (2003); K. Dimopoulos, Phys. Rev. D 68, 123506 (2003); K. Dimopoulos, G. Lazarides, D. Lyth, and R. Ruiz de Austri, J. High Energy Phys. 05 (2003) 057; M. Endo, M. Kawasaki, and T. Moroi, Phys. Lett. B 569, 73 (2003); S. Kasuya, M. Kawasaki, and F. Takahashi, Phys. Lett. B 578, 259 (2004); K. Hamaguchi, M. Kawasaki, T. Moroi, and F. Takahashi, Phys. Rev. D 69, 063504 (2004); D. H. Lyth, Phys. Lett. B 579, 239 (2004); J. McDonald, Phys. Rev. D 69, 103511 (2004); A. Mazumdar, hep-th/ 0310162; M. Giovannini, Phys. Rev. D 69, 083509 (2004); K. Dimopoulos, G. Lazarides, D. H. Lyth, and R. Ruiz de Austri, Phys. Rev. D 68, 123515 (2003).

[16] Super-Kamiokanade Collaboration, S. Fukuda et al., Phys. Rev. Lett. 86, 5656 (2001); SNO Collaboration, Q. R. Ahmad et al., Phys. Rev. Lett. 87, 071301 (2001).

[17] Super-Kamiokanade Collaboration, S. Fukuda et al., Phys. Rev. Lett. 85, 3999 (2000).

[18] M. Gell-Mann, P. Ramond, and R. Slansky, in Supergravity, edited by P. van Niewenhuizen and D. Z. Freedman (North-Holland, Amsterdam, 1979); T. Yanagida, in Proceedings of Workshop on Unified Theory and Baryon Number in the Universe, edited by O. Sawada and A. Sugamoto (KEK, Japan, 1979); R. N. Mohapatra and G. Senjanović, Phys. Rev. Lett. 44, 912 (1980).

[19] M. Fukugita and T. Yanagida, Phys. Lett. B 174, 45 (1986); Phys. Rev. D 42, 1285 (1990).
[20] A. Mazumdar, Phys. Rev. Lett. 92, 241301 (2004); A. Mazumdar, Phys. Lett. B 580, 7 (2004).

[21] V. A. Rubakov, Phys. Usp. 44, 871 (2001) [Usp. Fiz. Nauk 171, 913 (2001)].

[22] L. Randall and R. Sundrum, Phys. Rev. Lett. 83, 4690 (1999).

[23] L. Randall and R. Sundrum, Phys. Rev. Lett. 83, 3370 (1999).

[24] S. L. Dubovsky, V. A. Rubakov, and P. G. Tinyakov, Phys. Rev. D 62, 105011 (2000).

[25] P. Binetruy, C. Deffayet, and D. Langlois, Nucl. Phys. B565, 269 (2000); J. M. Cline, C. Grojean, and G. Servant, Phys. Rev. Lett. 83, 4245 (1999).

[26] R. N. Mohapatra, A. Pérez-Lorenzana, and C. A. de S. Pires, Int. J. Mod. Phys. A 16, 1431 (2001).

[27] C. Barceló and M. Visser, Phys. Lett. B 482, 183 (2000).

[28] A. Hebecker and J. March-Russell, Nucl. Phys. B608, 375 (2001).

[29] W. Muck, K. S. Viswanathan, and I.V. Volovich, Phys. Rev. D 62, 105019 (2000); R. Gregory, V. A. Rubakov, and S. M. Sibriyakov, Classical Quantum Gravity 17, 4437 (2000).

[30] A. Mazumdar, Phys. Rev. D 64, 027304 (2001); Nucl. Phys. B597, 561 (2001).

[31] R. Allahverdi, A. Mazumdar, and A. Pérez-Lorenzana, Phys. Lett. B 516, 431 (2001).

[32] W. D. Goldberger and M. B. Wise, Phys. Rev. D 60, 107505 (1999).

[33] See, for instance, the review by R. N. Mohapatra, hep-ph/ 0402035, and references therein.

[34] H. S. Goh, R. N. Mohapatra, and S. P. Ng, Phys. Lett. B 570, 215 (2003); Phys. Rev. D 68, 115008 (2003).

[35] A. D. Linde, Particle Physics and Inflationary Cosmology, Contemporary Concepts in Physics Vol. 5 (Harwood, Chur, Switzerland, 1990), p. 362.

[36] V.F. Mukhanov, H. A. Feldman, and R. H. Brandenberger, Phys. Rep. 215, 203 (1992).

[37] J. M. Bardeen, Phys. Rev. D 22, 1882 (1980); J. M. Bardeen, P. J. Steinhardt, and M. S. Turner, Phys. Rev. D 28, 679 (1983); J. M. Bardeen, Report No. DOE/ER/ 00423-01-C8.

[38] H. Kodama and M. Sasaki, Prog. Theor. Phys. Suppl. 78, 1 (1984).

[39] C. Gordon, D. Wands, B. A. Bassett, and R. Maartens, Phys. Rev. D 63, 023506 (2001).

[40] D. H. Lyth, C. Ungarelli, and D. Wands, Phys. Rev. D 67, 023503 (2003).

[41] D. N. Spergel et al., Astrophys. J. Suppl. Ser. 148, 175 (2003).

[42] M. Postma and A. Mazumdar, J. Cosmol. Astropart. Phys. 01 (2004) 005.

[43] J. Ellis, J. E. Kim, and D.V. Nanopoulos, Phys. Lett. B 145, 181 (1984); S. Sarkar, Rep. Prog. Phys. 59, 1493 (1996); M. Kawasaki and T. Moroi, Prog. Theor. Phys. 93, 879 (1995); A. L. Maroto and A. Mazumdar, Phys. Rev. Lett. 84, 1655 (2000); R. Kallosh et al., Phys. Rev. D 61, 103503 (2000).

[44] H. Murayama, H. Suzuki, T. Yanagida, and J. Yokoyama, Phys. Rev. Lett. 70, 1912 (1993).

[45] L. Covi, E. Roulet, and F. Vissani, Phys. Lett. B 384, 169 (1996). 